\title{
A second-order maximum entropy model predicts correlated network states, but not their evolution over time Aonan Tang${ }^{1}$, Jon Hobbs ${ }^{1}$, Wei Chen ${ }^{1}$, David Jackson ${ }^{2}$, Jodi L Smith ${ }^{3}$, Hema Patel ${ }^{3}$ and John M Beggs*1
}

\author{
Address: ${ }^{1}$ Indiana University Department of Physics, Bloomington, IN, 47405, USA, ${ }^{2}$ Brown University, Providence, RI, 02912, USA and ${ }^{3}$ Indiana \\ University Purdue University Medical School, Indianapolis, IN, 46202, USA \\ Email: John M Beggs* - jmbeggs@indiana.edu \\ * Corresponding author
}

from Sixteenth Annual Computational Neuroscience Meeting: CNS*2007

Toronto, Canada. $7-12$ July 2007

Published: 6 July 2007

BMC Neuroscience 2007, 8(Suppl 2):P32 doi:10.1 | 86//47|-2202-8-S2-P32

(C) 2007 Tang et al; licensee BioMed Central Ltd.

Highly correlated network states are often seen in multielectrode data, yet are predicted to be rare by independent models. What can account for the abundance of these multi-neuron firing patterns? Recent work $[1,2]$ has shown that it is possible to predict over $90 \%$ of highly correlated network states, even when correlations between neuron pairs are weak. To make these predictions, both groups used a maximum entropy model that fit only the firing rates and the pairwise correlations (a second-order maximum entropy model), and which was maximally uncommitted about all other model features. This new work raises several questions. First, how general are these results? Both previous reports largely used retinal data. Could this maximum entropy approach also succeed when applied to cortical slices? Although the original model explained correlations among spikes, could it also be used to explain the abundance of correlated states of local field potentials (LFPs)? A second issue concerns the abundance of correlated states over time. Can a secondorder maximum entropy model predict sequences of correlated states?

To examine the generality of this approach, we applied a second-order maximum entropy model to a variety of in vitro cortical networks, including acute slices from rat (n $=3$ ) and human epileptic tissue $(\mathrm{n}=1)$, as well as organotypic $(\mathrm{n}=3)$ and dissociated cultures $(\mathrm{n}=3)$ from rat.
We explored its effectiveness in predicting correlated states of both spikes and LFPs at one time point. On average, the model accounted for $90 \pm 6 \%$ (mean \pm s.d.) of the available multi-information, in good agreement with previous studies. In all cases, the maximum entropy model significantly outperformed an independent model, demonstrating its effectiveness in explaining correlated states in cortical spikes and LFPs at one time point. We also explored how well the maximum entropy model predicted sequences of correlated states over time. Here, the model often failed to account for the observed sequence lengths. In 8/10 preparations, the distribution of observed sequences was significantly longer $(p \leq 0.003)$. We conclude that a second-order maximum entropy model can predict correlated states, but not their evolution over time. This suggests that higher-order maximum entropy models incorporating temporal interactions will be needed to account for the sequences of correlated states that are often observed in the data.

\section{Acknowledgements}

This work was supported by NSF grant number 0343636 to JMB.

\section{References}

I. Schneidman E, Berry MJ, Segev R, Bialek W: Weak pairwise correlations imply strongly correlated network states in a neural population. Nature 2006, 440(7087): I007-I0I2.

2. Shlens J, Field GD, Gauthier JL, Grivich MI, Petrusca D, Sher A, Litke AM, Chichilnisky EJ: The structure of multi-neuron firing patterns in primate retina. J Neurosci 2006, 26(32):8254-8266. 